\title{
Proficiency testing of drug susceptibility results in the Canadian Network of Public Health TB laboratories
}

\section{A LASZlo PhD, NATIONAL. TUBERCULOSIS LABORATORY NETWORK*}

A fter many years of steady decline in developed countries mainly because of improved socioeconomic conditions and effective control programs, tuberculosis (TB) cases began to level off or increase in the mid-1980s (1). The reasons for this reversal are numerous; the abandonment of TB control programs, increasing poverty and homelessness, drug addition, human immunodeficiency virus infection, immigration from high prevalence countries and outbreaks among native communities are some of the most important $(2,3)$.

Drug-resistant $\mathrm{TB}$, in particular multidrug-resistant $\mathrm{TB}$ (MDRTB), poses a major threat to TB control efforts. Recent and well-publicized outbreaks in the United States and in some European countries have raised alarm levels (4,5). Effective surveillance of drug-resistant TB requires reliable epidemiological data and high quality laboratory testing of TB drug resistance.

Proficiency testing is an essential part of any quality assurance program in a clinical laboratory setting (6). Proficiency testing of TB drug susceptibility testing is assured internation-

${ }^{*}$ Mabel Rodrigues, Vancouver, British Columbia; Sylvia Chomyc, Edmonton, Alberta; Edward Chan, Regina, Saskatchewan; Dorothy Cheke, Saskatoon, Saskatchewan; Joyce Wolfe, Winnipeg, Manitoba; Albert Haddad, Etobicoke, Ontario; Louise Thibert, Sainte-Annede-Bellevue, Quebec; Mona Crowley, Saint John, New Brunswick, David Haldane, Halifax, Nova Scotia; Sandra March, St John's, Newfoundland

National Reference Centre for Tuberculosis, Bureau of Microbiology, Laboratory Centre for Disease Control, Ottawa, Ontario

Correspondence and reprints: Dr A Laszlo, National Reference Centre for Tuberculosis, Laboratory Centre for Disease Control, EHC Building No 8, Room 230, Tunney's Pasture, Address Locator 0802D, Ottawa, Ontario KIA OL2. Telephone 613-957-1819, fax 613-941-4546,e-mail Adalbert_Laszlo@isdtcp3.hwc.ca ally by a network of Supranational Reference Laboratories (SRL) set up by the World Health Organization (WHO) and the International Union against Tuberculosis and Lung Disease (IUATLD) in 1994 (7). The network comprises 20 reputable laboratories, located on five continents, and assures interlaboratory quality control of drug susceptibility testing within the network by ensuring uniformity and reproducibility of results obtained and their international comparability. Reference panels of strains of Mycobacterium tuberculosis are regularly distributed among the network laboratories by the coordinating laboratory, the National Reference Centre for Tuberculosis (NRCT) of the Laboratory Centre for Disease Control in Ottawa, Ontario, a WHO Collaborating Centre for Tuberculosis Bacteriology Research. The analysis of results obtained after three rounds of proficiency within the SRL network suggested that, at least for isoniazid and rifampin, reliable results can be obtained and compared internationally.

As part of its recently renewed mandate, the NRCT has begun coordinating a proficiency testing program of TB drug susceptibility testing within the Network of Provincial Public Health TB Laboratories in Canada, integrating this program with the proficiency testing conducted through the SRL network. This report summarizes the results obtained from the first round of national proficiency testing of TB drug resistance results, undertaken in 1996.

\section{METHODOLOGY}

Isoniazid, rifampin, ethambutol, streptomycin and pyrazinamide were tested. Identical sets of 10 clinical isolates of $M$ tuberculosis in duplicate (20 cultures) were sent to the laboratories of the network. This panel of cultures was identical to that used for the first round of proficiency testing within the SRL network. All but one province participated in this first 


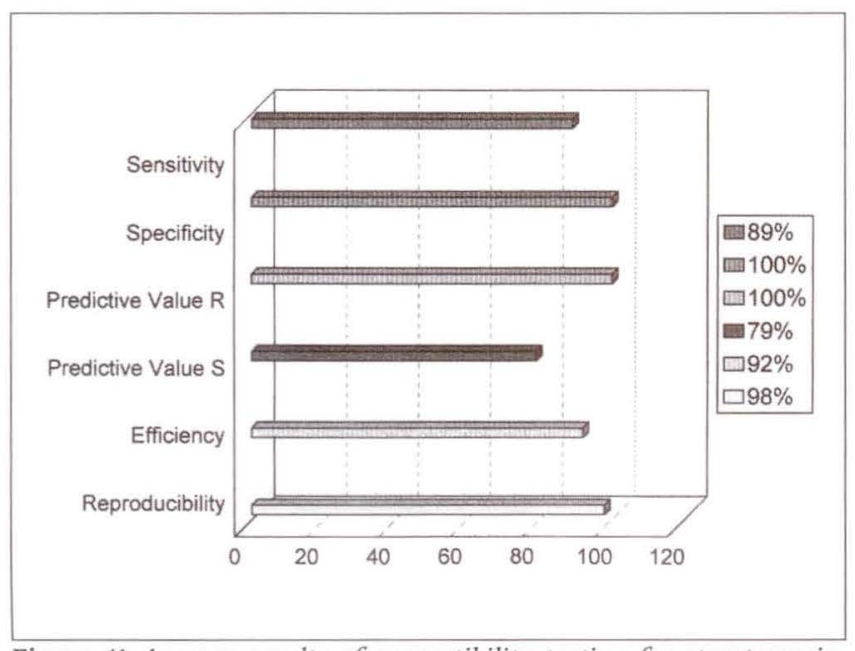

Figure 1) Average results of suspectibility testing for streptomycin, Proficiency Test Round 1

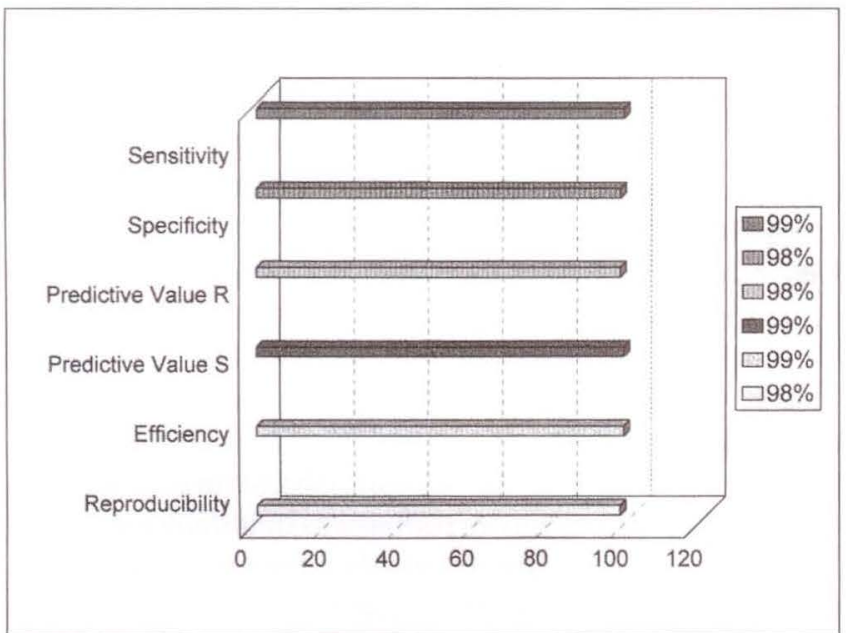

Figure 2) Average results of suspectibility testing for isoniazid, Proficiency Test Round 1

round of testing, and one of the provinces was represented by two laboratories. Each site received an identifier known only to the individual laboratory and NRCT. The cultures were identified with randomly chosen numbers that varied from site to site. The culture panel included three pairs of drug susceptible isolates - one of which was reference strain $\mathrm{H} 37 \mathrm{Rv}$ - and seven pairs of drug-resistant isolates encompassing some of the most commonly encountered resistance marker combinations, ie, two pairs of streptomycin-resistant isolates, two pairs of isoniazid/streptomycin-resistant isolates, two pairs of isoniazid/streptomycin/ethambutol-resistant isolates and one pair of isoniazid/streptomycin/rifampin/pyrazinamide-resistant isolates.

All laboratories used the proportion method-based radiometric BACTEC 460 (Becton Dickinson Diagnostic Systems, Maryland) procedure. All laboratories were to adhere strictly to the detailed procedures described in the BACTEC 460 product and procedures manual. All laboratories used the same concentrations of isoniazid, rifampin and pyrazinamide: 0.1 $\mu \mathrm{g} / \mathrm{mL}, 2.0 \mu \mathrm{g} / \mathrm{mL}$ and $100.0 \mu \mathrm{g} / \mathrm{mL}$, respectively. The concen-

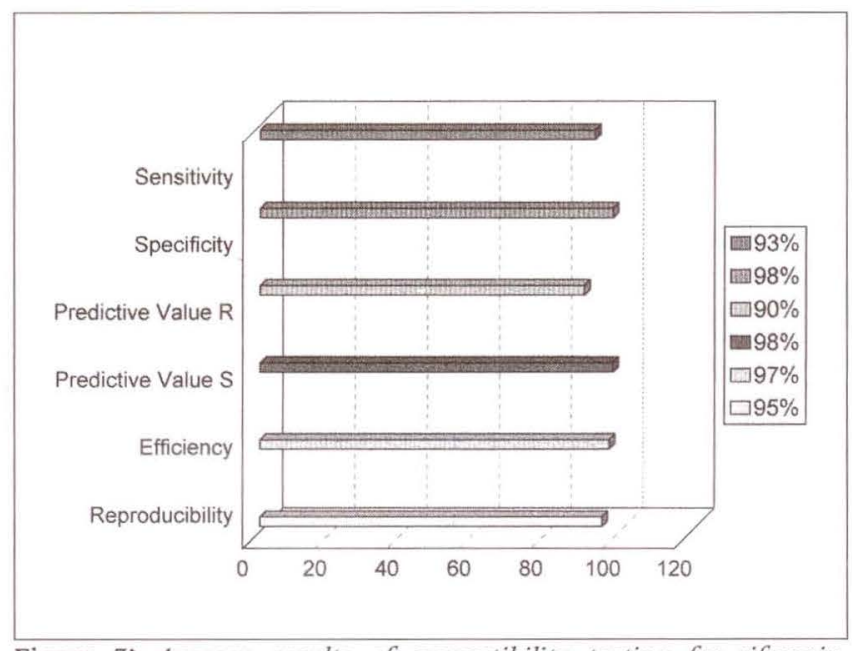

Figure 3) Average results of suspectibility testing for rifampin, Proficiency Test Round 1

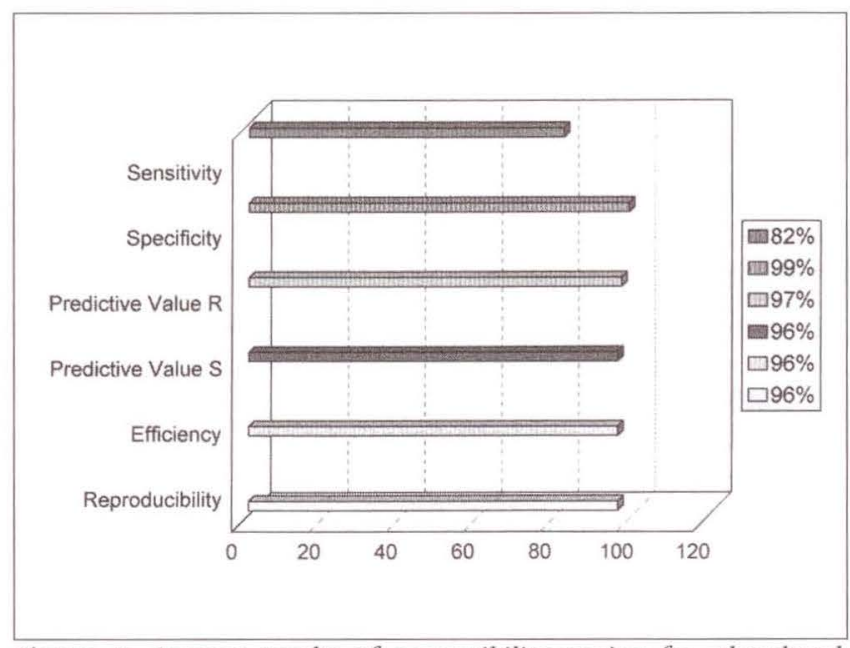

Figure 4) Average results of suspectibility testing for ethambutol, Proficiency Test Round 1

tration used for streptomycin was $2.0 \mu \mathrm{g} / \mathrm{mL}$ at all sites, except two where $4.0 \mu \mathrm{g} / \mathrm{mL}$ and $6.0 \mu \mathrm{g} / \mathrm{mL}$ were used. The concentrations used for ethambutol varied from $2.5 \mu \mathrm{g} / \mathrm{mL}$ to $7.5 \mu \mathrm{g} / \mathrm{mL}$. Results were to be interpreted by the participating laboratories as described in the BACTEC manual. Cultures were classified as resistant or susceptible. Laboratories reported results to the NRCT for collation and analysis.

The sample size of 20 cultures was determined to yield a significance level of alpha equal to 0.05 , allowing the detection of a true difference between laboratory methods with a power of $90 \%$. Results from all laboratories were compared with the judicial results obtained in the first round of testing within the WHO/IUATLD SRL Network, specifically the agreement of the majority of the participating laboratories which was considered the 'gold standard'.

A program for Bayesian analysis designed with Lotus 123 version 4 (Lotus Development Corporation, Massachusetts) was used to interpret the data. This analysis yields values for sensitivity, specificity and efficiency, as well as predictive values for resistance and susceptibility (8). Intralaboratory 
agreement between duplicate cultures was expressed as percentage reproducibility. Results were mailed to the participating laboratories.

\section{RESULTS}

Streptomycin: Figure 1 shows the overall analysis of the proficiency testing results for streptomycin. Average specificity of testing and, consequently, average predictive value for resistance were $100 \%$. Average sensitivity of testing was $89 \%$ (range $43 \%$ to $100 \%$ ), and average predictive value for susceptibility was $79 \%$ (43\% to $100 \%$ ). Average efficiency was $92 \%$ (60\% to $100 \%$ ), and average reproducibility $98 \%$ ( $80 \%$ to $100 \%$ ). Isoniazid: Figure 2 shows the overall analysis of the proficiency testing results for isoniazid. Average specificity of testing was $98 \%(89 \%$ to $100 \%)$ and the average predictive value for resistance was $98 \%$ (90\% to $100 \%$ ). Average sensitivity of testing was $99 \%$ ( $90 \%$ to $100 \%$ ), and the average predictive value for susceptibility was $99 \%$ ( $91 \%$ to 100$)$. Average efficiency was $99 \%$ (94\% to $100 \%$ ) and average reproducibility was $98 \%$ ( $80 \%$ to $100 \%)$.

Rifampin: Figure 3 shows the overall analysis of the proficiency testing results for rifampin. Average specificity of testing was $98 \%$ (93\% to $100 \%$ ), and average predictive value for resistance was $90 \%(67 \%$ to $100 \%)$. Average sensitivity of testing was $93 \%$ $(50 \%$ to $100 \%)$, and average predictive value for susceptibility was $98 \%$ ( $89 \%$ to $100 \%$ ). Average efficiency was $97 \%$ ( $89 \%$ to $100 \%$, and average reproducibility was $95 \%(60 \%$ to $100 \%$ ). Ethambutol: Figure 4 shows the overall analysis of the proficiency testing results for ethambutol. Average specificity of testing was $99 \%$ ( $93 \%$ to $100 \%$ ), and average predictive value for resistance was $97 \%$ ( $75 \%$ to $100 \%$ ). Average sensitivity of testing was $82 \%(0 \%$ to $100 \%)$ and average predictive value for susceptibility was $96 \%(80 \%$ to $100 \%)$. Average efficiency was $96 \%(80 \%$ to $100 \%$ ), and average reproducibility was $96 \%$ (60\% to $100 \%)$.

Pyrazinamide: Figure 5 shows the overall analysis of the proficiency testing results for pyrazinamide. Average specificity of testing and consequently the average predictive value for resistance were $100 \%$. Average sensitivity of testing was $65 \%$ ( $0 \%$ to $100 \%)$, and average predictive value for susceptibility was $92 \%(80 \%$ to $100 \%)$. Average efficiency was $93 \%(80 \%$ to $100 \%$, and average reproducibility was $97 \%$ ( $80 \%$ to $100 \%)$.

\section{REFERENCES}

1. Brancker A. Tuberculosis in Canada, 1989. Health Rep 1991;3:1.

2. Selwyn PA, Hartel D, Lewis VA, et al. A prospective study of the risk of tuberculosis among intravenous drug abusers with human immunodeficiency virus infection. N Engl J Med 1989;320:545-50.

3. Enarson DA, Grzybowski S. Incidents of active tuberculosis in the native populations of Canada. Can Med Assoc J 1976;134:1149-52.

4. Edlin BR, Tokars JI, Grieco MH, et al. An outbreak of multidrug resistance tuberculosis among hospitalized patients with the acquired immunodeficiency sysndrome. $\mathrm{N}$ Engl J Med 1992;326:1514-21.

5. Pearson ML, Jereb JA, Frieden TR, et al. Nosocomial transmission of multidrug-resistant Mycobacterium tuberculosis: a risk to

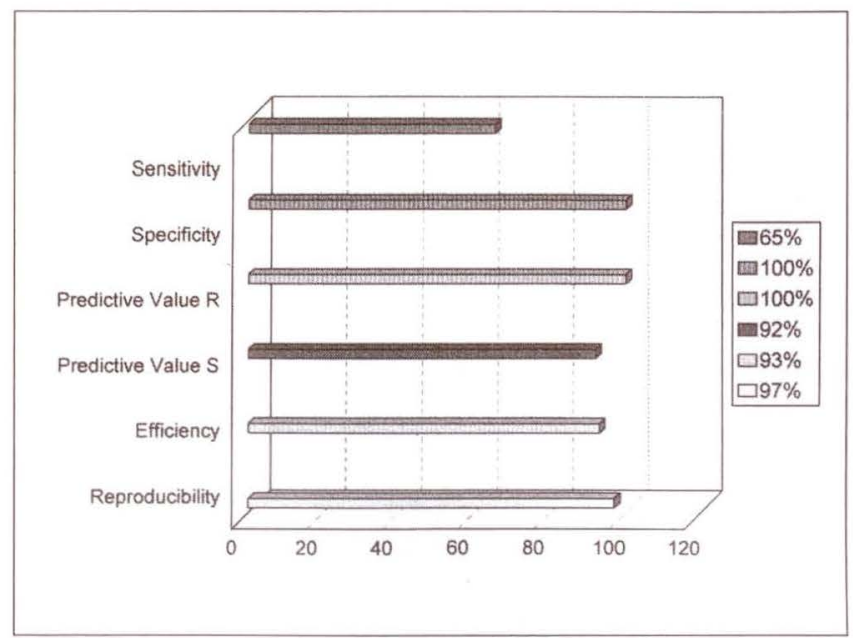

Figure 5) Average results of suspectibility testing for pyrazinamide, Proficiency Test Round 1

\section{CONCLUSIONS}

Drug susceptibility results obtained in the first round of proficiency testing within the national network of TB laboratories show that the highest sensitivity and specificity levels were reached in the testing of isoniazid and rifampin, the two drugs that define MDRTB today. The sensitivity of the testing was lower for streptomycin and ethambutol, and lowest for pyrazinamide. Some of the discrepancies seen in the latter results could be due to changes in the critical drug concentrations used in some of the network laboratories. It is worth noting that the specificity of testing was uniformly high for all five drugs tested. The average values for sensitivity and specificity, predictive values for susceptibility and resistance as well as efficiencies were not significantly different from those obtained in the first round of proficiency testing within the WHO/IUATLD SRL network. Thus, the average drug susceptibility testing of $M$ tuberculosis in public health laboratories in Canada compares well with that of some of the most reputable TB laboratories worldwide. Resistance to isoniazid and rifampin is detected with a high degree of proficiency across the country, a circumstance that augurs well for the success of MDRTB surveillance and TB control in Canada.

patients and health care workers. Ann Intern Med 1992;117:191-6.

6. Woods GL, Ridderhof JC. Quality assurance in the mycobacteriology laboratory, quality control, quality improvement, and proficiency testing. Clin Mycobacteriol 1996; 16:657-75.

7. Bustreo F, Migliori GB, Nardini S, Raviglione MC. Antituberculosis drug resistance: Is it worth measuring? The WHO/IUATLD Working Group on Antituberculosis Drug Resistance Surveillance. Monaldi Arch Chest Dis 1996:51:299-302.

8. Toman K. Sensitivity, specificity and predictive value of diagnostic tests. Bull Int Union Tuber 1981;56:18-28. 


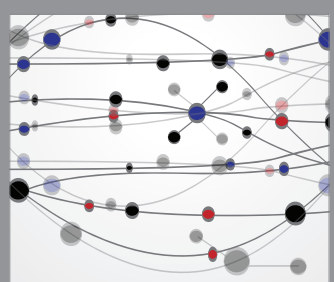

The Scientific World Journal
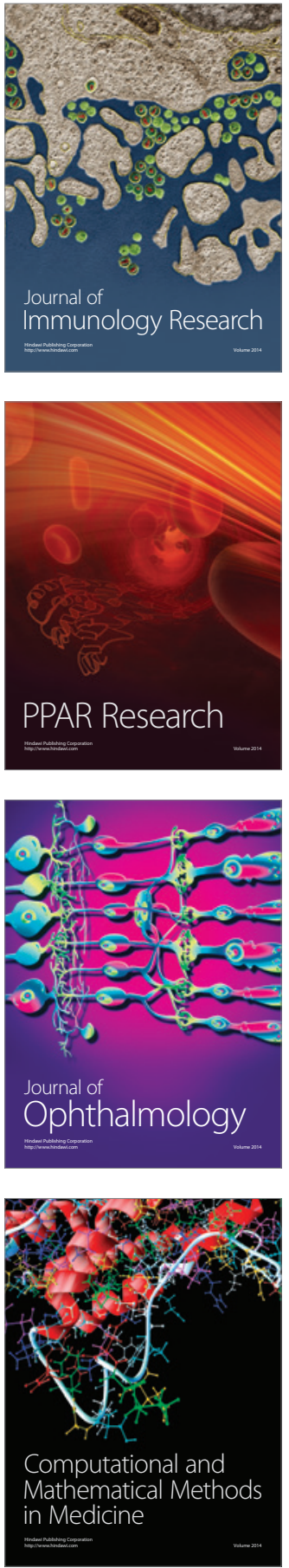

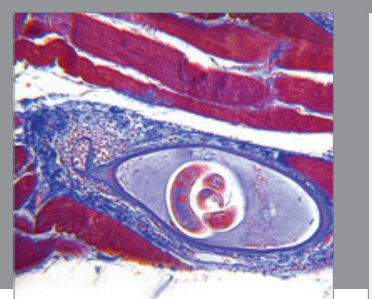

Gastroenterology Research and Practice

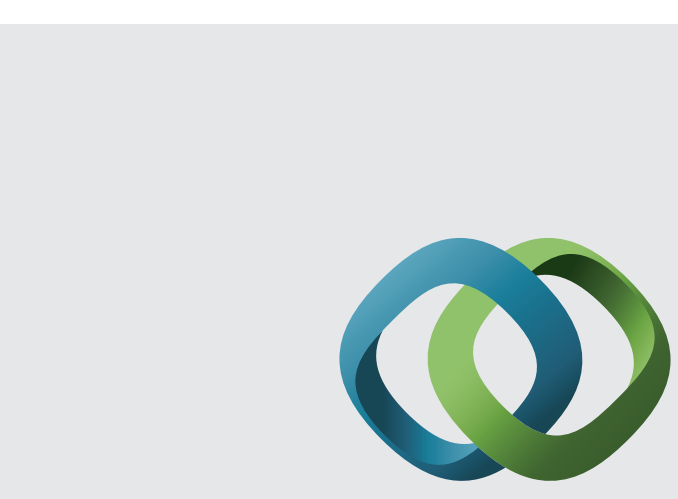

\section{Hindawi}

Submit your manuscripts at

http://www.hindawi.com
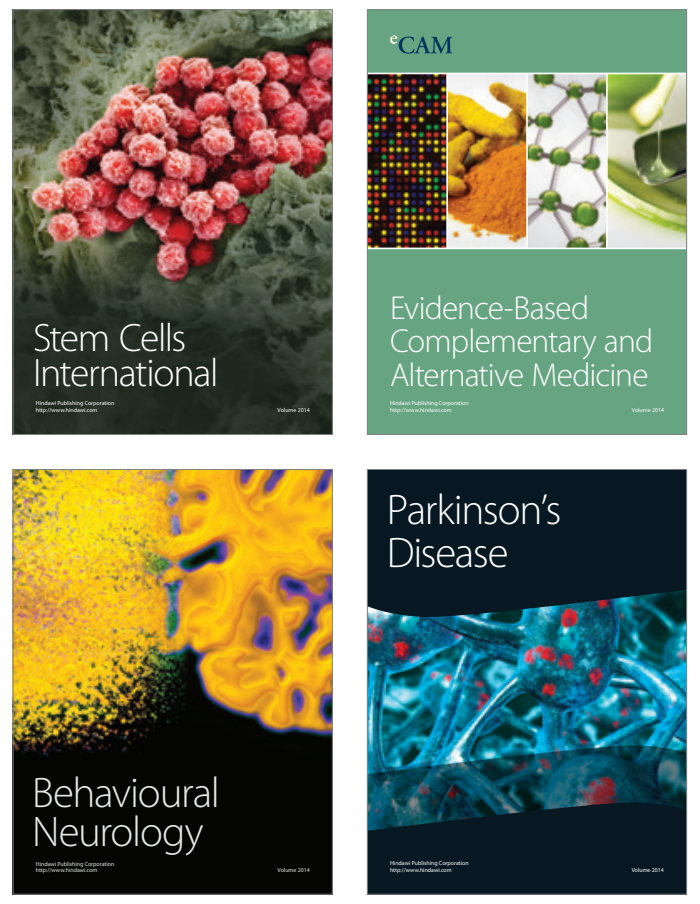
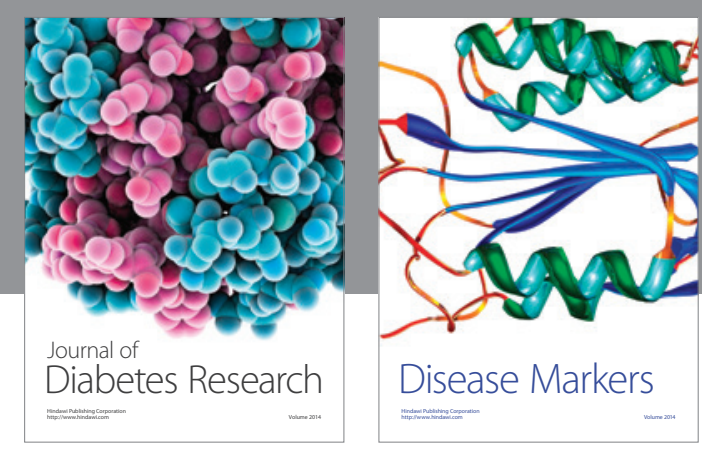

Disease Markers
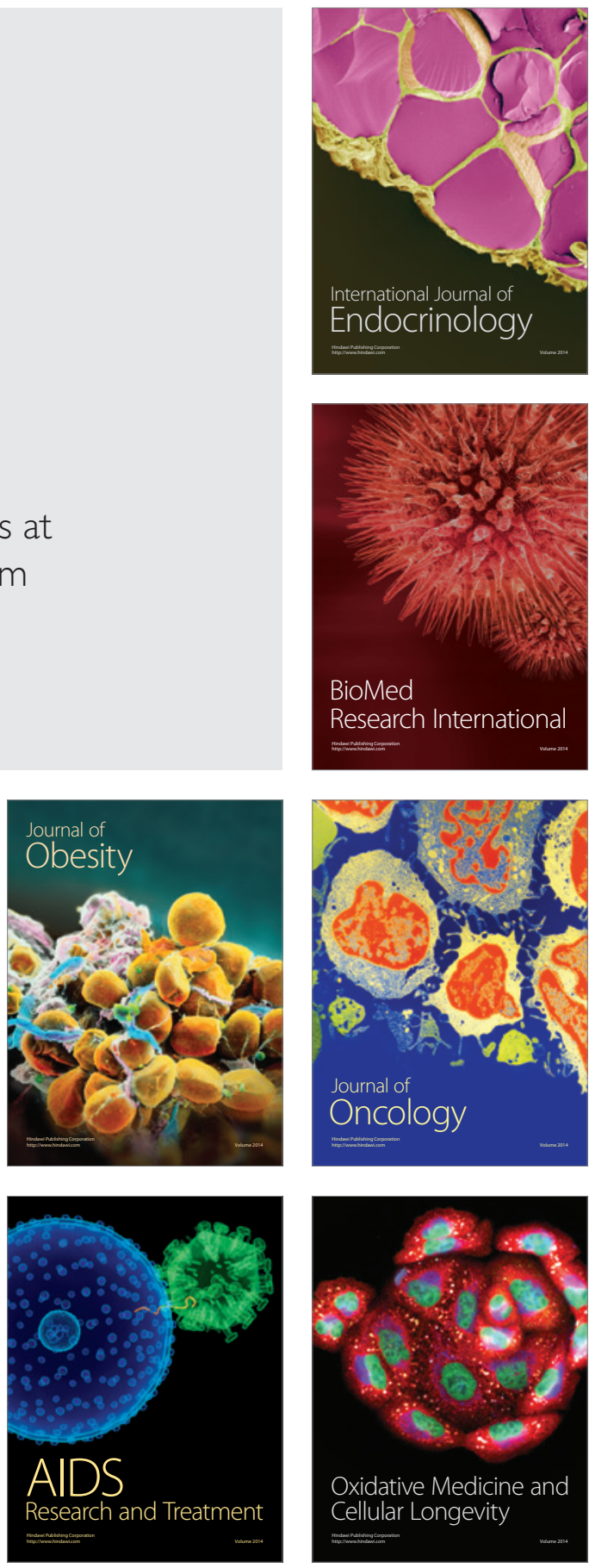